\title{
KNITTING TECHNOLOGIES AND TENSILE PROPERTIES OF A NOVEL CURVED FLAT-KNITTED THREE-DIMENSIONAL SPACER FABRICS
}

\author{
Xiaoying $\mathrm{Li}^{1,2}$, Gaoming Jiang ${ }^{1 *}$, Xiaolin $\mathrm{Nie}^{1}$, Pibo $\mathrm{Ma}^{1^{*}}$, Zhe Gao ${ }^{1}$ \\ ${ }^{1}$ Engineering Research Center for Knitting Technology, Jiangnan University, Wuxi 214122, China; \\ ${ }^{2}$ School of Art and Design, Guangdong University of Technology, Guangzhou 510090, China \\ E-mail: Jiang G. jiang@526.cn Ma P. mapibo@jiangnan.edu.cn
}

\begin{abstract}
:
This paper introduces a knitting technique for making innovative curved three-dimensional (3D) spacer fabrics by the computer flat-knitting machine. During manufacturing, a number of reinforcement yarns made of aramid fibres are inserted into $3 D$ spacer fabrics along the weft direction to enhance the fabric tensile properties. Curved, flat-knitted 3D spacer fabrics with different angles (in the warp direction) were also developed. Tensile tests were carried out in the weft and warp directions for the two spacer fabrics (with and without reinforcement yarns), and their stress-strain curves were compared. The results showed that the reinforcement yarns can reduce the fabric deformation and improve tensile stress and dimensional stability of $3 D$ spacer fabrics. This research can help the further study of $3 D$ spacer fabric when applied to composites.
\end{abstract}

\section{Keywords:}

Three-dimensional spacer fabric, aramid fibre, computer flat-knitting machine, knitting technology, tensile property

\section{Introduction}

Textile structure is widely used in reinforcement composites, such as weaving and braiding textiles combined with epoxy for some reinforcement composites in aerospace, luxury car industry. This is due to the fact that textile structure can maximise the materials mechanical properties during the application. Therefore, new textile structures have attracted the attention of more and more researchers for exploration of textile composites. In recent years, flat-knitted three-dimensional (3D) spacer fabrics have been becoming attractive for making composites due to the specific properties. The structure of flat-knitted 3D spacer fabric is novel it is made of two separate fabric layers connected vertically by pile yarns. These 3D spacer fabrics normally have high thickness and excellent performance, such as good structural integrity, low weight and density, and high impact resistance, heat resistance and sound absorption. These structural 3D fabrics have found uses in transport vehicles, aircraft, storage, bridge, marine applications and others [1-6]. The separated layers of flat-knitted 3D spacer fabrics can be connected by pile yarns or fabric layers. The spacer fabrics bound with fabric layers have greater potential applications and development value because of the fabric without limited thickness from the distance between two needle beds. Flat-knitted 3D spacer fabrics could be manufactured using the advanced modern flat-knitting machine, which may be a good solution to develop such innovative 3D structures. The unique technical features herein include transfer, racking, goring, multi-gauges, and so on [4, 6-10]. At present, studies of spacer fabrics are mostly based on weaving and warp knitting but not flat knitting [11-14]. The materials are more concentrated on glass and carbon fibre owing to their high modulus and high coefficient of friction $[11,12]$. Studies are paying more attention to fabric without curvature [11-16]; only a few studies have been conducted on the curvilinear fabrics [1-3].
On comparing with glass and carbon fibres, aramid fibres and high-strength, high-modulus polyethylene fibres have better weaving performance. It is known that glass and carbon are used extensively due to their high modulus and high tensile strength, but they also show high stiffness, low bending strength and high knitting difficulty at the same time, which induce lower productive efficiency. Therefore, aramid fibres are preferred for the development of 3D spacer fabrics.

One goal of the current study is to develop curvilinear flatknitted 3D spacer fabrics by using aramid fibres. These fabrics can be used as reinforcing materials for different composites with improved mechanical properties. This can meet the high requirements of industrial production. In order to achieve this objective, a few actual spacer fabrics were manufactured by using aramid fibres. In view of the technology, curved flat-knitted 3D spacer fabrics in different angles (in the warp direction) were produced through the recently developed computerised flat-knitting machine. Reinforcement yarns are inserted into 3D spacer fabrics in the weft direction to enhance the fabric tensile properties. A further fundamental analysis of 3D spacer fabric composites is compared the results of tensile properties of two structure fabrics (with and without reinforcement yarns).

\section{Experimental}

\subsection{Flat-knitted innovative 3D spacer fabrics}

\subsubsection{Materials}

Aramid fibres (440Dtex/100f $\times 3$ ) were used in knitting that were supplied by Sinopec Yizheng Chemical Fibre Company Limited in Jiangsu, China. 


\subsubsection{Computerised flat-knitting machine}

Knitting was carried out by a modern computerised flat-knitting machine (CMS530 of company STOLL from Germany) as shown in Figure 1. Gauge was $3.5 \mathrm{~mm}$. With a gauge range from $E 5$ to $E 18$, a working width of $50 \mathrm{in} / 127 \mathrm{~cm}$ and three systems, the CMS 530 can knit complex patterns quickly and is highly productive. It enables an optimal combination of 'knit/ transfer/knit' with structured, distribution and racking patterns, which makes it ideal for high-level, flexible production and extremely short setup and adjustment times.

\subsubsection{Flat-knitted 3D spacer fabrics}

Computerised flat-knitting technique is a better way of developing innovative structures by using unique technical

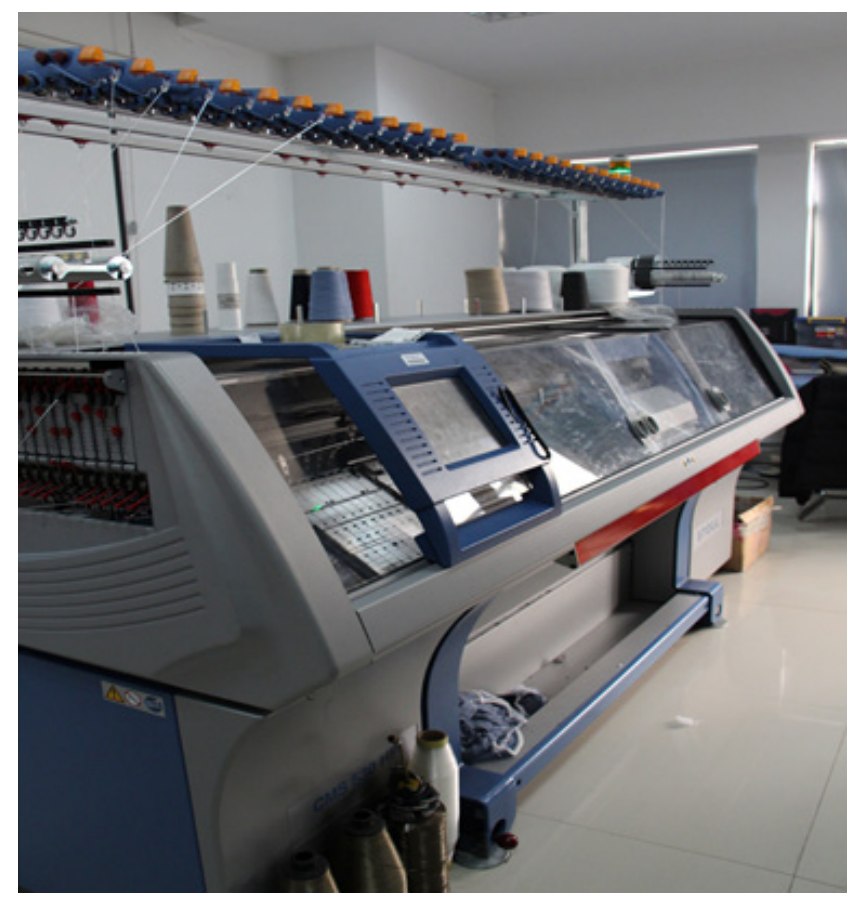

(a) STOLL computer flat knitting machine

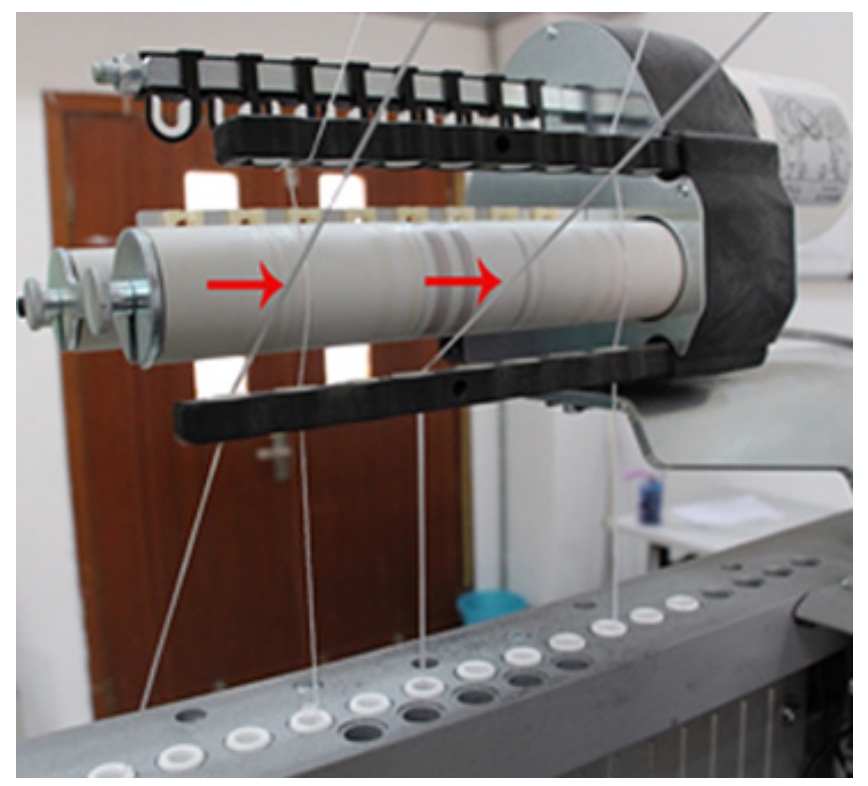

(b) Simplified yarn path

Figure 1. Knitting on STOLL CMS530 HP features, such as holding down sinkers, transfer, racking, and so on. Spacer fabrics show complex 3D constructions with two separate fabric layers bonded to each other. The yarns have to travel a long way from the yarn feeder to the knitting area, which produces large friction and big input tension. Therefore, a simplified yarn path as shown in Figure 1 to ensure smooth knitting is very important. And it is useful to turn down the comb pull, especially in the case of narrow knitting area. The technological basis of manufacturing such innovative 3D spacer fabrics is illustrated in Figures 2-5.

(a). Single jersey is knitted separately on front and back needle beds $(A 1, A 2)$ until the height of the requirement $(H) P 1$ and $P 2$ are arrived at.

(b). Single jersey is knitted one by one separately on both needle beds (L1, L2) until half of the height of the requirement.

(c). The connection is done with rib courses by one by one needle selection using the same yarns.

(d). Single jersey is knitted one by one separately again on both needle beds (L3, L4), back to P1, P2.

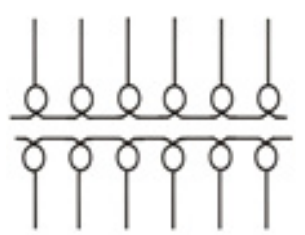

(a)

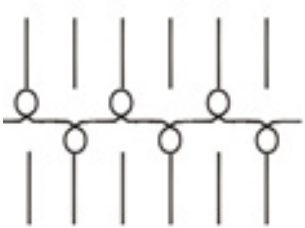

(c)

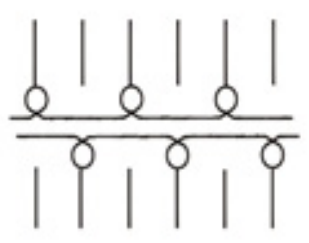

(b)

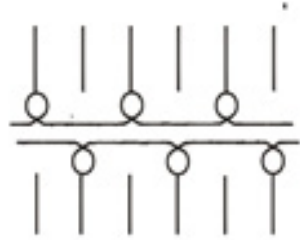

(d)
Figure 2. Knitting flow chart of flat-knitted 3D spacer fabrics

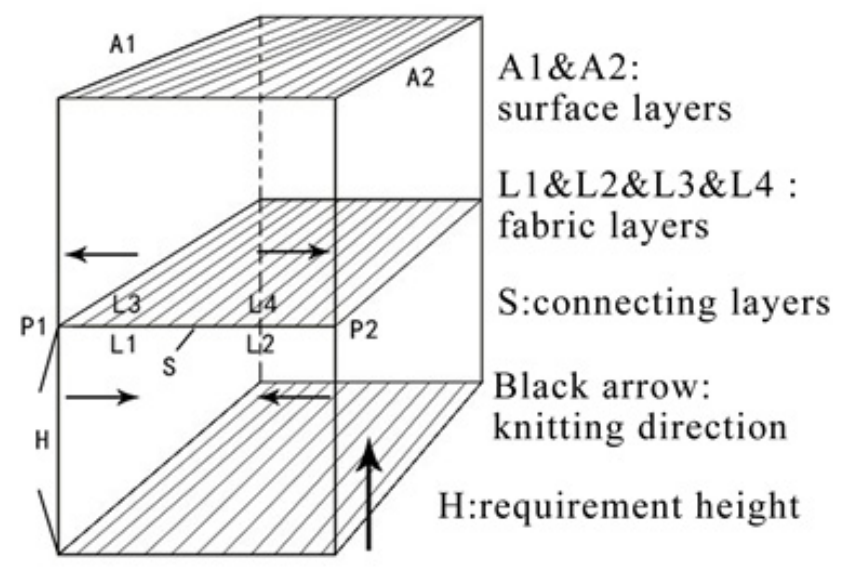

Figure 3. Diagram of flat-knitted 3D spacer fabrics 
The flat-knitted 3D spacer fabrics are developed based on the above-mentioned innovative knitting skill. In our previous work, rib course has been done only once in connecting layer. However, during experiments, it was found that a single rib was too easy to bend to meet the requirement. Therefore, the program was improved to keep the space shape better by knitting rib course twice.

\subsubsection{Development of flat-knitted 3D spacer fabrics with reinforcement yarns}

In order to enhance the tensile properties of flat-knitted 3D spacer fabrics, the reinforcement yarns are integrated into 3D spacer fabrics in surface layers or in fabric layers (in the weft direction). If the reinforcement yarns are replaced by functional or conductive yarns (such as carbon filament, copper wire) in flat-knitted 3D spacer fabrics. If the reinforcement yarns are replaced by other high-functioning fibres, the flat-knitted 3D spacer fabrics could be used as high-functioning fabrics. The way reinforcement yarns are inserted into $3 D$ spacer fabrics in surface layers is shown in Figures 5-6.

(a). Single jersey is knitted separately by front and back needle beds.

(b). Float is knitted separately on front and back needle beds. Tuck could be done after am interval length in order to avoid float line too long.
(Step a alternates with step b until the height of the requirement. Steps c-e are the same as Steps b-d in Figure 2)

\subsubsection{Flat-knitted 3D curvilinear spacer fabrics}

The advanced flat-knitting technique for 3D spacer fabrics also permits the various curvilinear shapes in the warp direction. The curvilinear spacer is an innovative structure, which can be developed out of flat-knitted 3D spacer fabrics. To maintain the relative balance of comb-down, the length of $\mathrm{H}$ is changed, and the $L$ should be adjusted accordingly to ensure knit smoothly.

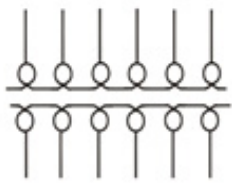

(a)

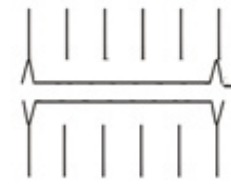

(b)

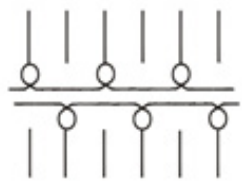

(c)

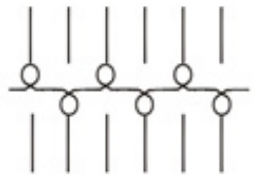

(d)

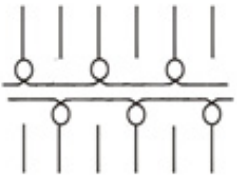

(e)
Figure 4. Knitting flowchart of 3D spacer fabrics with reinforcement yarns

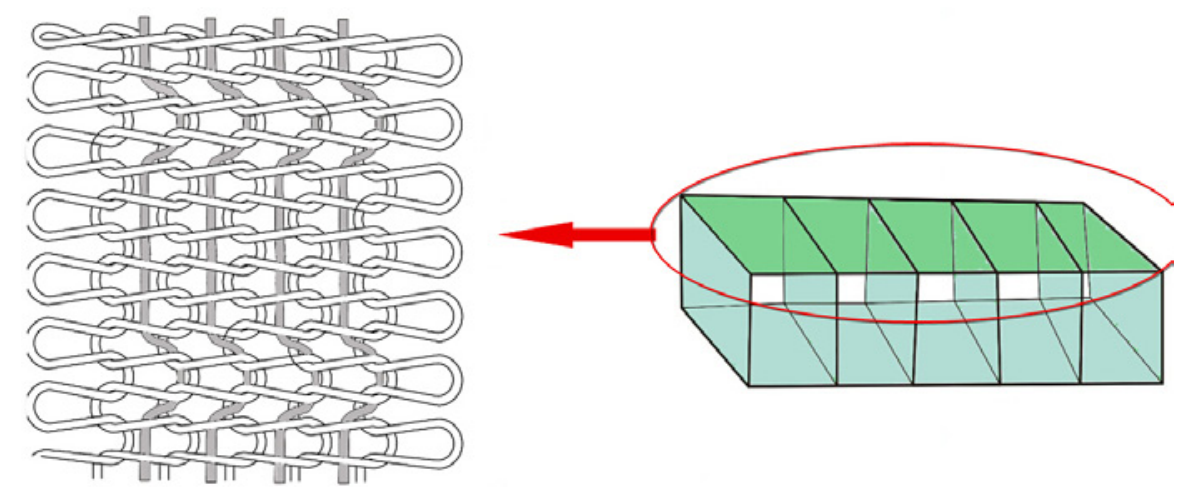

a) Knitting structure of top side of 3D spacer fabric

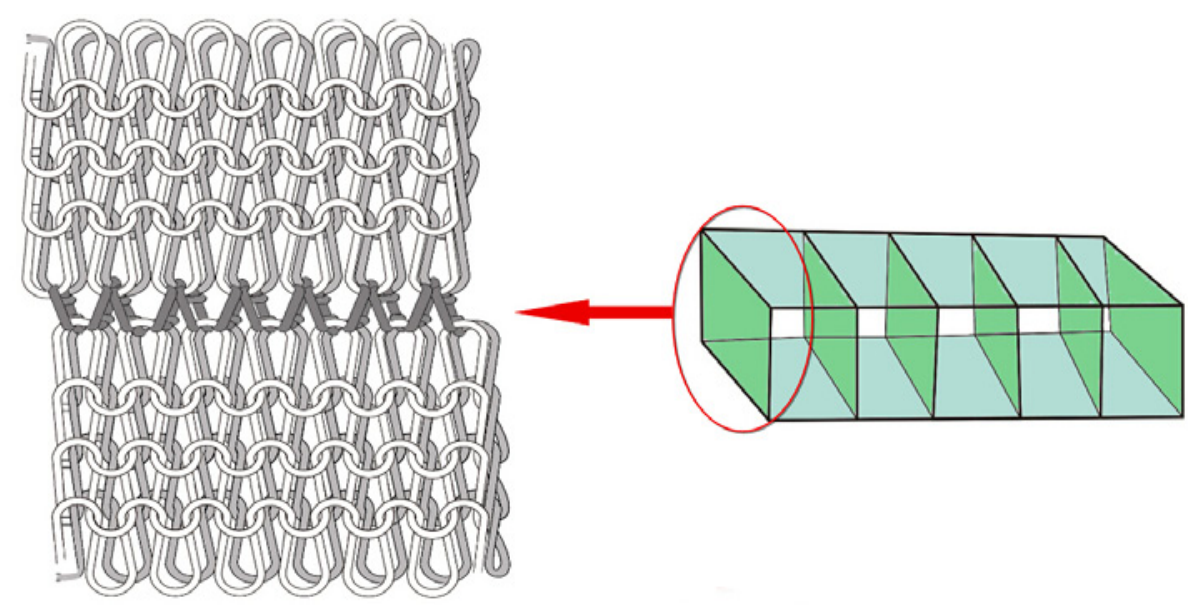

b) Knitting structure of left side of 3D spacer fabric

Figure 5. The knitting structures of 3D spacer fabric 


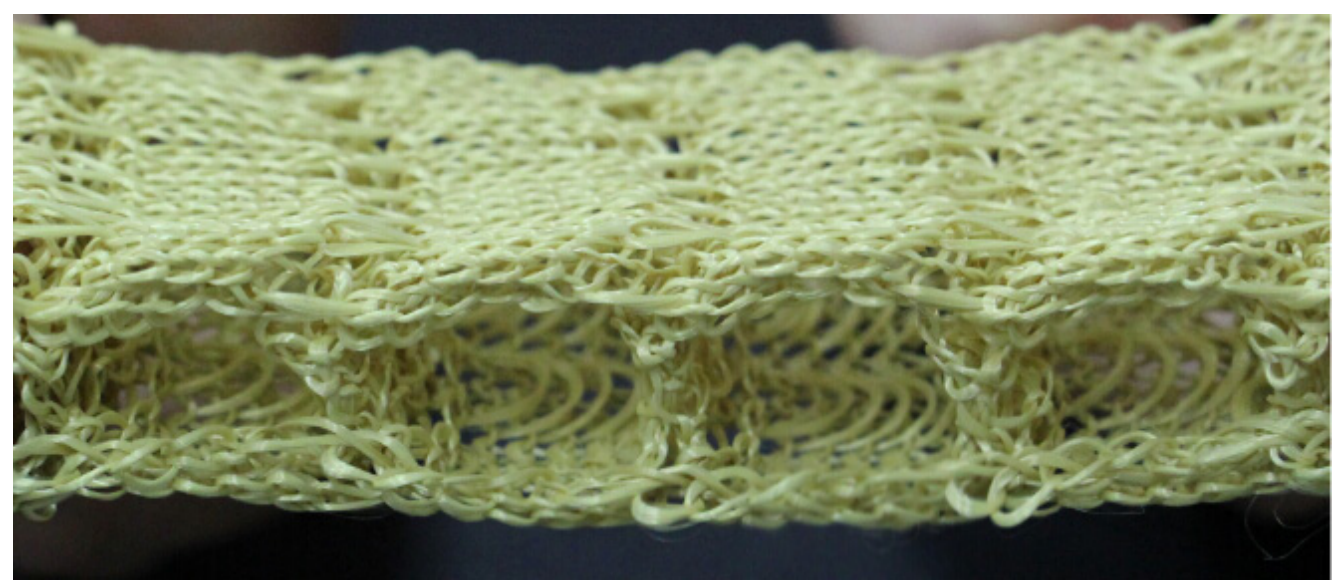

(a) With reinforced yarns

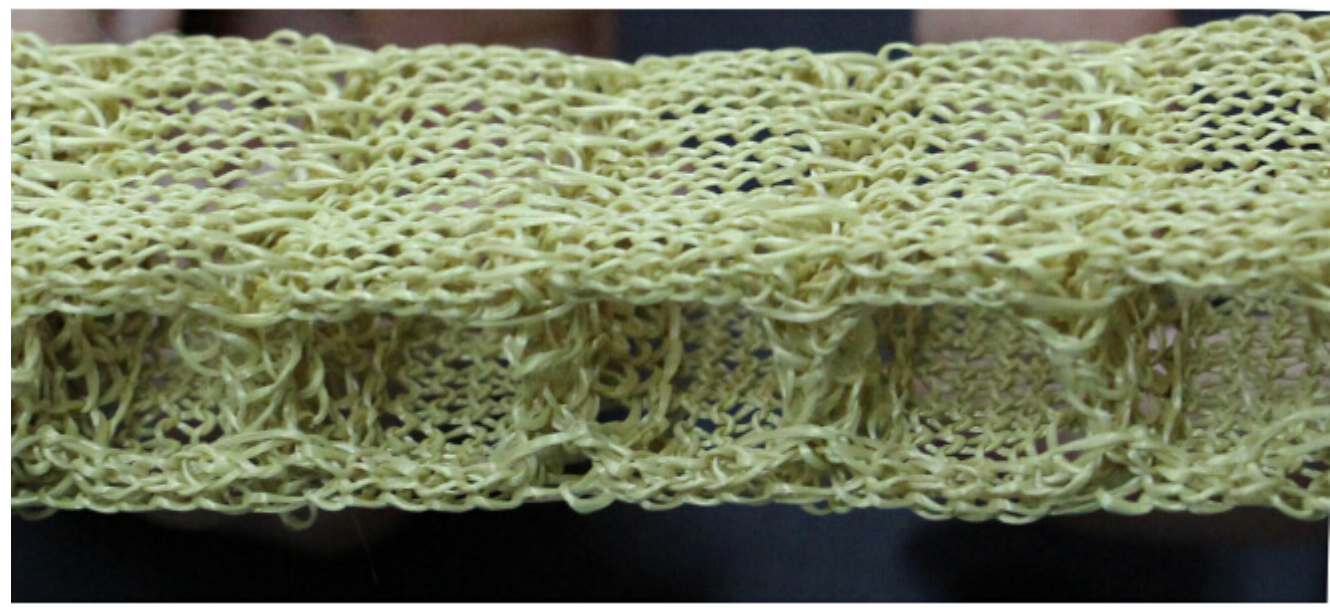

(b) Without reinforced yarns

Figure 6. Flat-knitted 3D spacer fabric with and without reinforced yarns

For instance, as it is shown in Figures 7-9, the length of $\mathrm{H} 1$ is shorter than $\mathrm{H} 2$, so the length of L1 and L3 are longer than L2 and $L 4$.

\subsection{Tensile properties of flat-knitted innovative 3D spacer} fabrics

\subsubsection{Sample preparation}

Fabric samples are $120 \mathrm{~mm} \times 50 \mathrm{~mm}$, which are shown in Figure 10. Two kinds of $0^{\circ}$ curved flat-knitted 3D spacer fabrics (with and without reinforcement yarns) are divided according to weft and warp directions. There are 12 pieces of samples for two directions. Each of the two ends of the samples were infiltrated by resin before test in order to ensure all the yarns bore stress evenly to avoid breaking at gripping points.

\subsubsection{Tensile testing of flat-knitted innovative 3D spacer fabrics}

The tensile tests are performed on an INSTRON universal material testing machine (Figure 11). Experimental conditions: Tensile speed is $10 \mathrm{~mm} / \mathrm{min}$, spacing length is $60 \mathrm{~mm}$, test temperature is $25^{\circ} \mathrm{C}$ and humidity is $50 \%$.

\section{Results and discussion}

\subsection{Innovative flat-knitted 3D spacer fabrics and curvilinear effects}

Shape stability is achieved by simply knitting the rib twice in fabric layers. This innovative flat-knitted $3 D$ spacer fabric shows better mechanical properties obviously. And then in order to enhance the tensile properties of such spacer fabrics, the reinforcement yarns are integrated in weft direction (Figure 8-a). The 3D curved spacer fabrics of arc angle in warp direction from $0^{\circ}$ to $360^{\circ}$ were realised successfully (Figure $8-b, 9)$. The distance between the surface layers and fabric layers was designed to be $15 \mathrm{~mm}$. Because of inclined joining by the fabric layers, it is dissimilar for both surface layers of the spacer fabrics with the curvature angles of $90^{\circ}$ and $360^{\circ}$.

\subsection{Tensile properties of 3D spacer fabrics with and without reinforcement yarns}

The typical stress-strain curves of samples in different directions are shown in Figure 12-a and 12-b, respectively. It can be seen from these figures that samples with reinforcement yarns show improved tensile properties in both the two directions. The 
beginning of the tensile curves is similar, but the differences become enhanced with the increase of tensile strain. The reason is, samples with reinforcement structures have lot of straight yarns that enhance the tensile properties obviously in weft direction. However, in warp direction, because of some overlapping coils from reinforcement yarns and surface layers, the tensile properties of fabrics with reinforcement yarns are still higher than fabrics without reinforcement yarns.

\section{Conclusion}

Three-dimensional spacer fabrics are produced innovatively and successfully by knitting rib course twice in fabric layers and inserting reinforcement yarns in surface layers (weft direction), and then with different angles (warp direction). All these fabrics are manufactured using aramid fibre by computerised flatknitting machine.

According to the results of stress-strain test, we came to the conclusion that the tensile properties of spacer fabrics with reinforcement yarns are superior (in both weft and warp directions) to fabrics without reinforcement yarns.

Therefore, beyond the traditional application trends in the fields of lightweight composite and integrated sandwich composites, this textile-reinforced structure can potentially be used in some industrial fields by filling in the wax, wires, stacking electronic chips, and embedded electronic components.

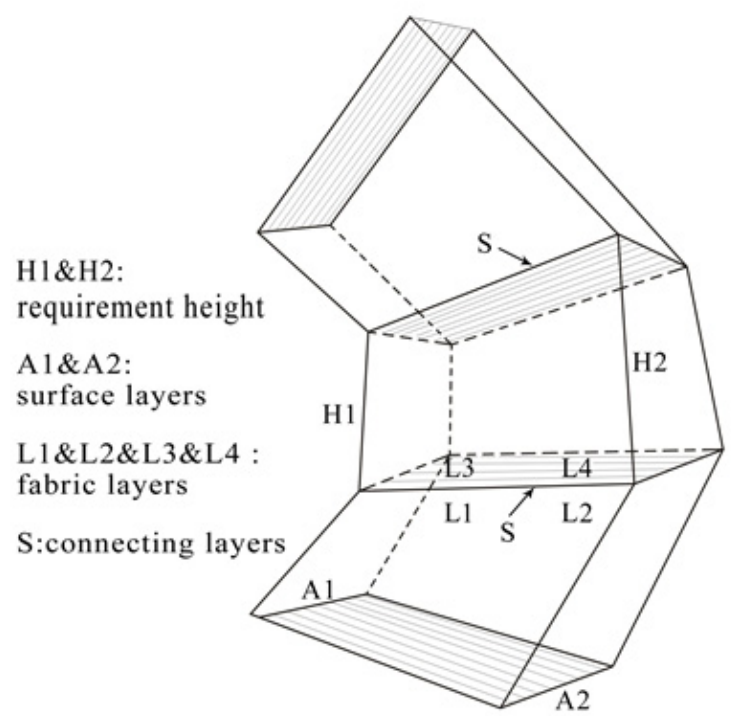

Figure 7. Diagram of flat-knitted 3D curvilinear spacer fabrics

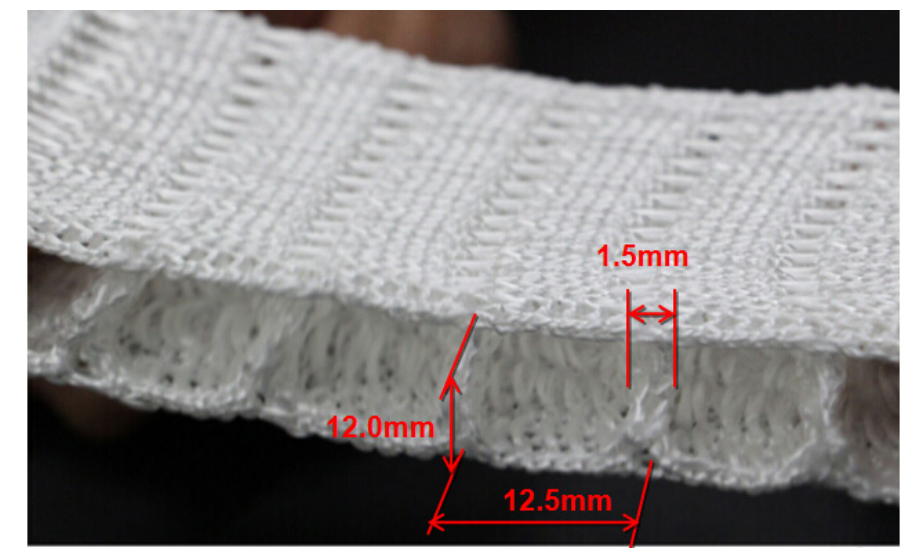

(a) Flat-knitted 3D spacer fabric with reinforced yarns

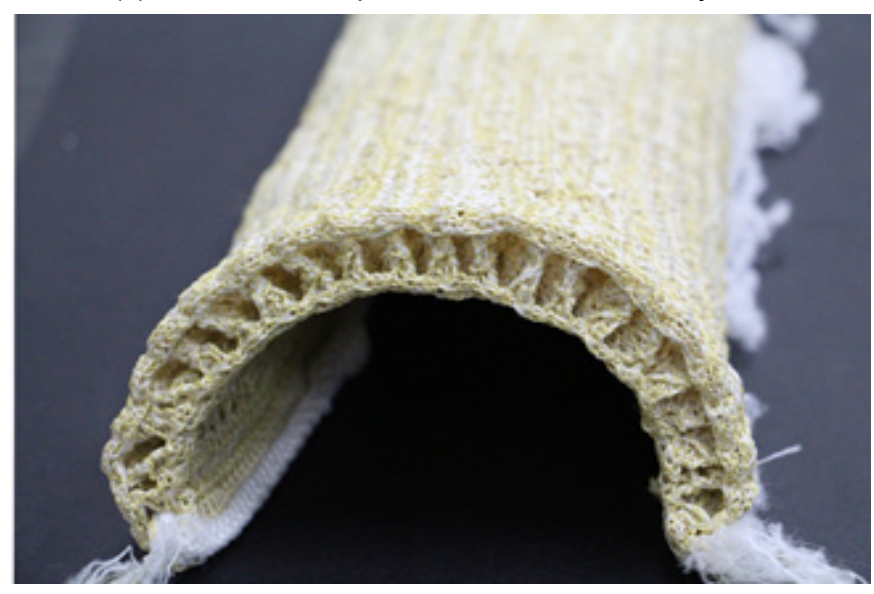

(b) Spacer fabric with $180^{\circ}$ curvature

Figure 8. Fabric samples
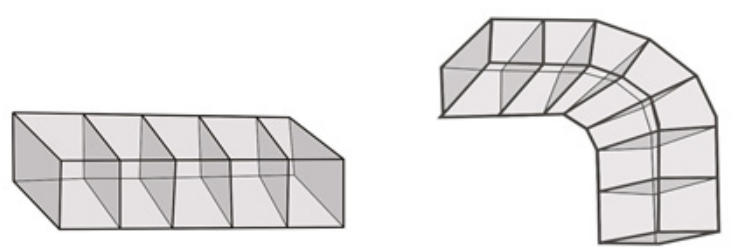

Curvature: $0^{\circ}$

Curvature: $90^{\circ}$

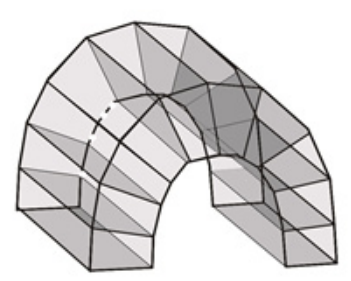

Curvature: $180^{\circ}$

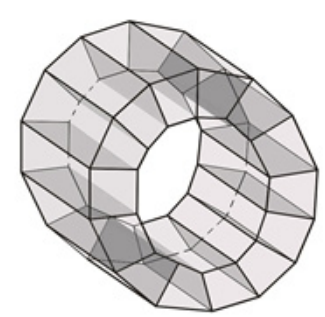

Curvature: $360^{\circ}$

Figure 9. Diagram of $3 \mathrm{D}$ spacer fabrics with different curvature angles

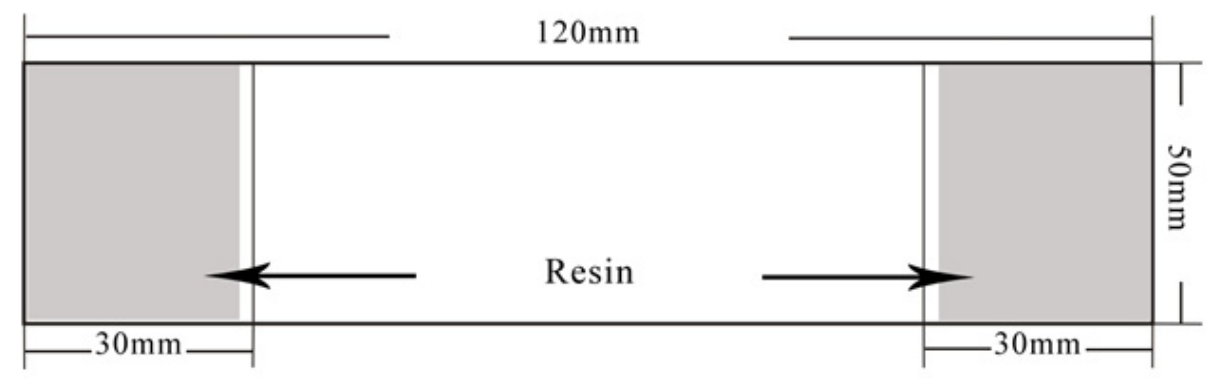

Figure 10. Fabric sample for the test 


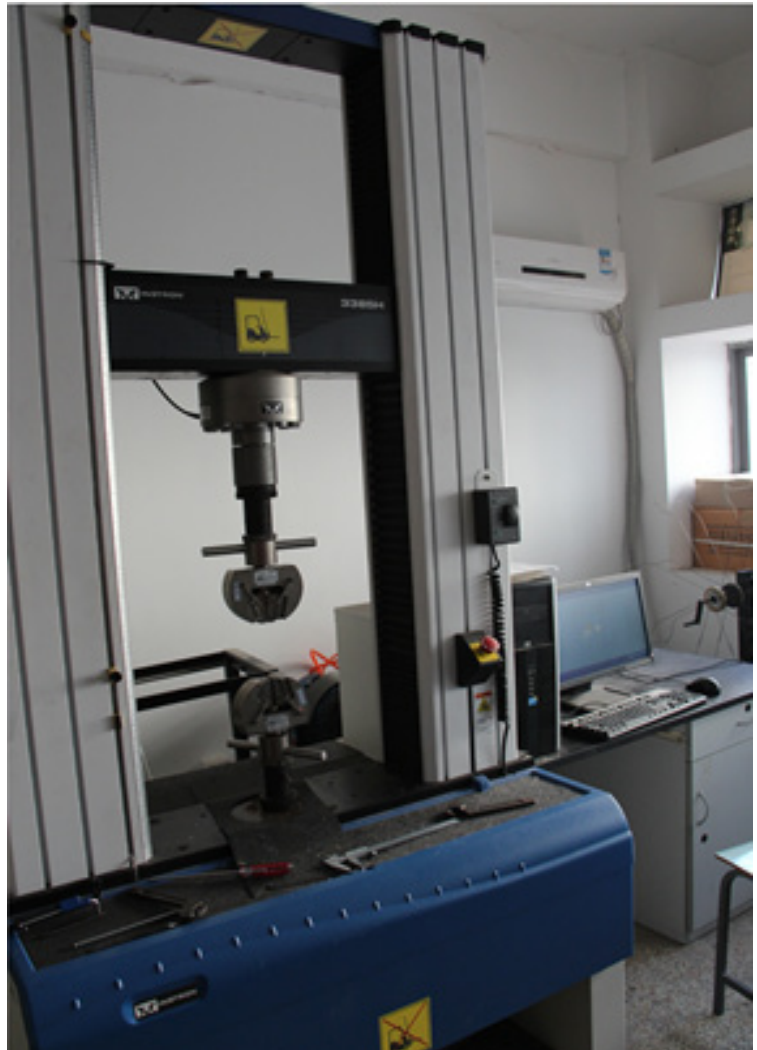

Figure 11. Instron universal material testing machine

\section{Acknowledgements}

The authors acknowledge the financial support from the National Science Foundation of China (No.11302085 and 51403080), the Fundamental Research Funds for the Central Universities (No. JUSRP1043 and JUSRP51404A), and the Innovation fund project of Cooperation among Industries, Universities \& Research Institutes of Jiangsu Province (No. BY2014023-34 and BY2014023-20).

\section{References}

[1] Abounaim MD and Chokri C. Flat-knitted innovative three-dimensional spacer fabrics: a competitive solution for lightweight composite applications. Textile Research Journal, 2012: 82(3): 288-298.

[2] Abounaim MD, Olaf $D$, Gerald $H$ and Chokri $C$. Thermoplastic composites from curvilinear $3 D$ multilayer spacer fabrics. Journal of Reinforced Plastics and Composites, 2010: 29(24): 3554-3565.

[3] Abounaim MD, Gerald $H$, Olaf $D$ and Chokri C. Thermoplastic composites from curvilinear flat-knitted $3 D$ multi-layer spacer fabric using hybrid yarn and the study of $2 D$ mechanical properties. Composites Science and Technology, 2010: 70: 363-370.

[4] Unal A, Hoffmann $G$ and Cherif C. Development of weft knitted spacer fabrics for composite materials. Melliand Textile Berichte, 2006: 4(224-226): E49-50.

[5] Abounaim MD. Modelling of technical bindings and manufacturing of flat knitted and woven spacer fabrics with hybrid (GF/PP) yarn as sandwich structure. Master's thesis No-1310, Department of Mechanical Engineering, Technische Universitat Dresden, Germany, 2006.

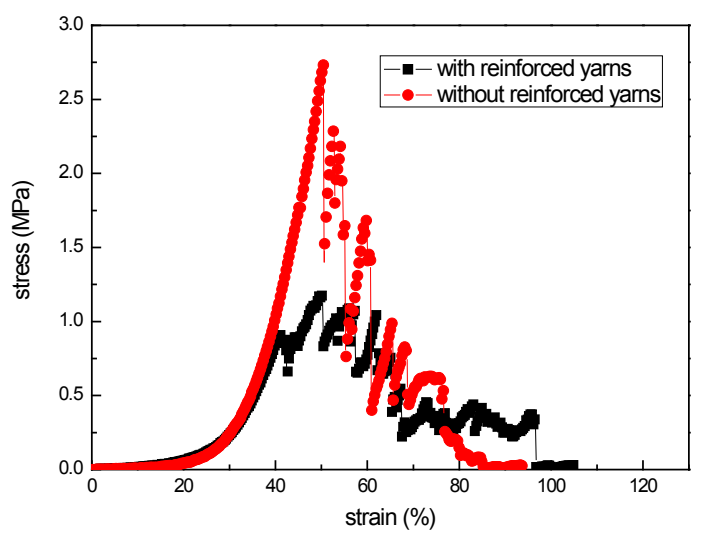

(a) Stress-strain relation of fabric under tensile behaviour along $0^{\circ}$ direction

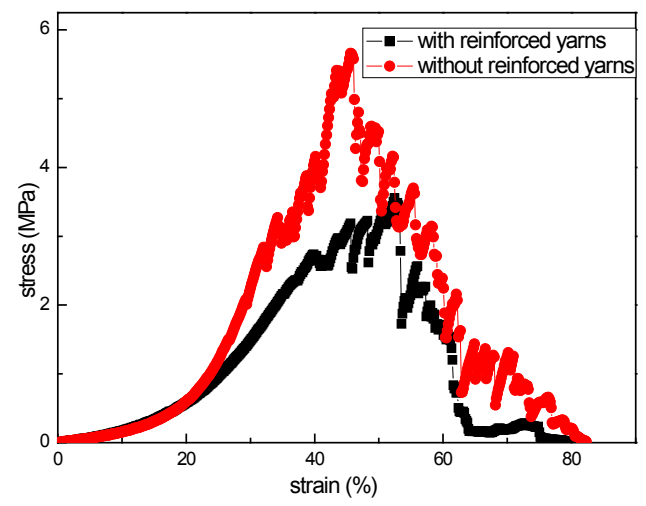

(b) Stress-strain relation of fabric under tensile behaviour along $90^{\circ}$ direction

Figure 12. Stress-strain curves of $3 D$ spacer fabrics with and without reinforced yarns

[6] Hu $H$, Araujo $M$ and Fangueiro R. 3D technical fabrics. Knitting Int, 1996: 1232: 55-57.

[7] Abounaim M, Hoffmann G, Diestel $O$ and Cherif C. 3D spacer fabric as sandwich structure by flat knitting for composites using hybrid yarn. In: AUTEX World Textile Conference, Izmir, Turkey, 26-28 May, 2009: 675-681.

[8] Araujo M, Hu H, Fangueiro R, Ciobanu O and Ciobanu L. Developments in weft-knitting technical textiles. In: 1st Autex Conference: TECHNITEX, Portugal, Vol. 1, 2001: 253-262.

[9] Ciobanu L. Developments on knitted sandwich fabrics with complex shapes. 1st Autex Conference: Technitex, Portugal, Vol. 1, 2001: 490-496.

[10] Abounaim M, Hoffmann G, Diestel O and Cherif C. Flatknitted spacer fabrics with hybrid yarns for composite materials. Melliand Textile berichte, 2009: 3-4(87-89): E30-E31.

[11] Cao H, Qian K, Wei Q and Li H. Low-velocity impact behaviour of 3-D glass fibre hollow integrated core sandwich composites. Polymers \& Polymer Composites, 2010: 18(4): 175-179.

[12] Cao H, Qian K, Wei Q and Li H. Compression after impact of 3-D integrated hollow core sandwich composites. Tekstil ve Konfeksiyon, 2011: 21(1): 16-21. 
[13] Ye X, Hu H and Feng X. Development of the warp knitted spacer fabrics for cushion application, Jounal of Industrial Textiles, 2008: 37(3): 213-223.

[14] Xie Y, Fangueiro R and Hu H. Application of warp-knitted spacer fabrics in car seats, Jounal of Textile Institute, 2007: 98(4): 337-343.

[15] Zhang M, Sun B, Hu H, Gu B. Dynamic behavior of 3D biaxial spacer weft-knitted composite T-beam under transverse impact. Mechanics of Advanced Materials and Structures, 2009: 16: 365-370.

[16] Zhang M, Hu H. Mechanical properties of novel different stitched density $3 D$ hybrid basalt composite. Proceedings of International Conference on Fibrous Materials, 2009: 12-16. 